\title{
III. DRIVE THEORY AND PRIMARY PROCESS: A PHILOSOPHICAL ACCOUNT
}

\author{
LINDA W. BRAKEL
}

\section{INTRODUCTION}

\section{1. DRIVES DEFINED AND DESCRIBED}

Drive theory in psychoanalysis concerns the "instinctual drives". Currently there is much controversy regarding the relation between instinctual drives and affects. Although for some theorists these are placed in a single category, as will be plain, the very philosophical analysis I am undertaking presupposes what amounts to a necessary and fundamental difference between drives and affects. Much of the account of drives provided in this article concerns the nature of drive objects; while clearly the very notion of an "affect object" is incoherent. Thus to my mind drives and affects must belong to two distinct categories. While this confounding of conceptual categories might be the latest problem drive theory is facing, it has not been its only one. At various times and in various quarters there have been great rhetorical debates and confusions about Freud's very use of the term. For the purpose of this article however (because the terminological issue is not relevant to the philosophical analysis) I will use what has become the standard reading (Nagera 1969, 1970) starting with Freud's (1905d) comments from Three Essays on Sexuality:

"By an ,instinct' [instinctual drive] is provisionally to be understood the psychical representative of an endosomatic [...] source of stimulation [...] the concept of instinct [instinctual drive] is thus one of those lying on the frontier between the mental and the physical. The simplest and likeliest assumption [...] would seem to be that in itself an instinct is without quality, and, so far as the mental life is concerned, only to be regarded as a measure of the demand made upon the mind for work. What distinguishes the instincts [instinctual drives] from one another and endows them with specific qualities is their relation to their somatic sources and to their aims " (Freud 1905d, p. 168).

After using almost identical words to define instinctual drives, Freud (1915c) in Instincts and Their Vicissitudes elaborated four terms needed to understand the concept. In addition to the "source" and "aims" 
of an instinctual drive, described in the earlier work, he added the "pressure" of a drive and its "object" (p. 122-3):

"By the pressure of an instinct we understand its motor factor, the amount of force or the measure of the demand for work which it represents [...] The aim of an instinct is in every instance satisfaction [...] obtained by removing the state of stimulation at the source of the instinct. But although the ultimate aim of each instinct remains unchangeable, there may yet be different paths leading to the same ultimate aim; so that an instinct may be found to have various nearer or intermediate aims [...] The object of an instinct is the thing [...] through which the instinct is able to achieve its aim. It is what is most variable about an instinct and is not originally connected with it, but becomes assigned to it only in consequence of being peculiarly fitted to make satisfaction possible. The object is not necessarily extraneous; it may equally well be a part of the subject's own body. It may be changed any number of times in the course of the vicissitudes which the instinct undergoes [...] It may happen that the same object serves for the satisfaction of several instincts simultaneously" (Freud 1915c, p. 122-3).

By the source of an instinct is meant the somatic process which occurs in an organ or part of the body and whose stimulus is represented in mental life by an instinct.

In sum then, a drive is the mental representative of a physiologic need state such that a certain pressure or quantitative demand for work, having a certain quality varying with particular drives, is placed on the psychological apparatus toward fulfillment and thereby cessation of the particular drive. Each drive in addition to its pressure, has a source (in the body), an aim (the distal part of which is always fulfillment, the proximal part varies with each drive and is the mode to achieve fulfillment), and an object. The object is that thing or those things or parts of things (which can include self or other or part thereof) through which satisfaction can be obtained. The source, aim, and object of a drive determine its qualitative nature. The pressure of a drive, mostly originating from its somatic source, constitutes the quantitative contribution.

\section{2. AN EXAMPLE: X'S ORAL DRIVES}

$\mathrm{X}$ is a baby, and $\mathrm{X}$ is hungry. $\mathrm{X}$ has a number of oral drives - to suck, swallow, and to feel the pleasure associated with pleasant taste and temperature and the comfort of gradual gastric fullness replacing empti- 
ness. The somatic sources are falling blood sugar, hypothalamic regulation and signaling, gastric stirrings, and sensations in the mouth. The aims are, distally to have this drive state reduced, and proximally to get fed. The object is whatever will satisfy these oral drives. Food not separable from whomever (or whatever or part of whomever or whatever) provides it, is an obvious object; but so is the pacifier, the rattle, and part of the blanket - whatever is good to suck on. Thus for Baby X the object of these oral drives is X's mother's left breast and nipple and her right breast and nipple and a bottle and nipple, and the milk, and her face, and how it feels being held and fed, and how her face and breast (or bottle) look, and the pacifier and the hand of its provider, and the blanket edge, especially how it feels in X's mouth - any part of this and all of it together.

\section{A PHILOSOPHICAL ANALYSIS OF DRIVES}

\section{1. DRIVES AND PROPOSITIONAL-ATTITUDE PSYCHOLOGY}

Drives do not fit well into the propositional-attitude psychology favored by philosophers to account for motivation and intention. Desires, in contrast, as the conative propositional attitude type often paired with beliefs (a cognitive propositional attitude type), fit readily. What is the difference? First here are some important similarities. Both drives and desires can be used in conjunction with behavior to infer the content of various cognitive propositional attitudes. For example, if $\mathrm{Mr}$. A says he desires a cool drink, then is seen walking toward a water-fountain, and then bends to drink, one can infer that he believes that water-fountains are sources of cool drinks and that he supposes (another cognitive propositional attitude) that this particular water-fountain is operational. Under some conditions the very same cognitive propositional attitude contents can be inferred from beliefs about the state of Mr. A's drives along with certain actions he performs, without his expressing a desire. Suppose, for example it is learned that Mr. A. has been deprived of fluids for some time. This state will, assuming Mr. A. is normal, lead to heightening of various oral drives and the very same inferences regarding the propositional attitudes associated with his ensuing acts follow. Conversely drives and desires can themselves be inferred given various cognitive propositional attitudes. Mr. A. starting to drink from a waterfountain usually implies that he believes that water-fountains are sources 
for cold water, and that he supposes that the particular water-fountain he is about to drink from works. From here it can be inferred that he is drinking because he desires a cool drink, likely in part owing to various oral drives that are active. Now this sort of belief/desire propositional attitude analysis can go wrong. Mr. A. can be a water-fountain inspector, with oral drives that are well satisfied, no desire whatever for cool drinks, and a belief that the water-fountain in question is in need of repair. But most of the time the sort of analysis performed on Mr. A. will go through, even dealing with drives. So what is the difference between drives and desires? And will we find that this difference accounts for why drives don't fit the standard propositional-attitude psychology analysis? The answer to the second question is yes.

Here is what is different. Whereas with any desire (or belief) its object can be picked out by a mental representation the content of which is determinate, no such singularly determinate content will do for the object of a drive. The representation of the objects of drives look radically indeterminate in content (see Velleman 2002, p. 90). Mr. A's desire for a cool drink has as its object "cool drink" and he has a mental representation of this. Whatever the nature of mental representations, Mr. A's mental representation of cool drink, refers singularly to cool drinks. Turning now to the object of Mr. A's oral drives, those likely driving his desire to seek a cool drink, there is no such straightforward analysis. The object of these drives, hence the content of the mental representation picking out the object, cannot be specified as singular and determinant, anymore in Mr. A's case than for Baby X above. The object can be approximated as some sort of amalgam of objects and parts of objects, all and whatever of which will bring forth a satisfied feeling in Mr. A's mouth. But any mental representation of this sort of drive object will seem "either radically indeterminate in content or as playing a nonstandard role" (Velleman 2002, p. 90). Velleman (2002, p. 91) describes such representations as "far too vague to be expressed in the concepts with which we consciously reason." He, in discussing the object of aggressive drives, continues (p. 91):

"If [...] we insist on framing a written or spoken 'that' clause to express the content of aggression, we shall have to concede that what the [aggressive drive] attitude can [...] be satisfied by [...] [is] not only the literal truth of the clause but also indefinitely many other outcomes related only by analogy, by metaphorical similarity, or by other mental associations of an open-ended variety." 
Velleman concludes (p. 91): "Either way, propositional-attitude psychology will not afford the same computational advantages in this case as it does in the case of ordinary beliefs and desires, whose tendencies [...] to be satisfied can be summed up in sentences of ordinary language."

\section{2. ARE DRIVE AIMS SINGULAR AND DETERMINATE?}

That drives have objects that cannot be represented in a univocal, determinant way is central to drives not admitting of a standard propositional-attitude psychology analysis. (More will be said about the nonsingular nature of drive objects in the next subsection.) But is the same absence of singular determinancy true of the aims of drives? In Freud's (1915c, p. 122) basic definitions, it is clear that the aim of any drive will consist of two parts: the "ultimate and unchangeable" aim of ceasing the state of stimulation through discharge and satisfaction, and the "different path" such that a drive may have "various nearer or intermediate aims". Given this compound aim for any drive, is there determinancy of the nearer and intermediate aims of a drive? Anna Freud (1936, p. 175) describes the aims of drives as easily modified, transformed, and displaced one for another. In a later work she states: "An individual who finds the path to an instinctual [drive] aim of adulthood blocked by internal or external obstacles can respond to such a frustration by reverting to childhood forms of satisfaction" (1977, p. 248). And she notes this can go in the opposite direction too: "under the influence of the superego, primitive [drive] impulses are transformed, inhibited in their aims, and the drive energies belonging to them deflected to moral, ethical, or socially higher aims." (p. 247). All of Freud's cases (See especially Dora in Freud 1905e; Little Hans in Freud 1909b; The Rat Man in Freud 1909d; and The Wolfman in Freud 1918b) show evidence of displaced aims. Further, his understanding of regression, symptom formation, and sublimation, despite other changes in his conceptions of these, all remained dependent on what for him was obvious: the aims of drives could be readily displaced (1900a, 1915c, 1915e, 1926d, 1940a).

Although it may seem that the foregoing suggests indeterminacy, I contend that in fact, the very transformability or displace-ability of the aims of drives, suggests that drives have original aims from which there can be displacement. There is support for this understanding from Freud (1905d, p. 168) in his Three Essays on Sexuality. In a passage stating 
that all instinctual drives have the distal aim of discharge and satisfaction, but differing proximal aims, he states that: "What distinguishes the instincts one from another and endows them with specific qualities is their relation to their somatic sources and to their aims." Discussing Freud's allusion to the "nearer and intermediate aims" Rapaport (1967, p. 831) comments: "Freud used the term 'aim' in two different senses. One of these is the tendency toward discharge; the other [...] is not the universal discharge tendency itself, but something more specific." The more specific quality of aims, is determined by the "instinctual mode" (according to Rapaport, p. 832, this is Erikson's concept; Erikson 1950), each mode associated with the source of an instinctual drive. Thus as a child gaining anal sphincter control is in the anal phase, the anal drives are associated with aims whose mode concern retaining and expelling. Anal phase drives of course far outlast the anal phase of development, and drives with aims to retain (money, for example) or expel (anger, troublesome others) are commonplace; but no moreso than displacements from these anal phase aims. For example the aim to retain (money) can be displaced onto the perhaps more acceptable aim of constructing a bank. Thus it is in the more specific sense of aim - the proximal aims with their particular instinctual modes - that there are original aims and then displacements from these originals. As Rapaport put it (p. 831): "Clearly, in this second sense of the term, the aim [my emphasis] can be restricted, displaced and substituted for."

\section{3. DRIVE OBJECTS ARE NOT SINGULAR. MUST THEY BE INDETERMINATE?}

The threat of indeterminancy for drive objects cannot be resolved so easily. While the case has been made for drives having original singular aims from which displacements occur, there is not a parallel case for drive objects. Returning to Freud's (1915c, pp. 122-3) definition of instinctual drive object, he states: "It is what is most variable about an instinct and is not originally connected with it, but becomes assigned to it only in consequence of being peculiarly fitted to make satisfaction possible." With drives possessing no specified original objects through which to gain discharge and satisfaction, in the role of drive object any person or material object or part thereof which fits a drive in order to make satisfaction possible is as good as any other person, material object, or part thereof.

Velleman (p. 90), in discussing his own aggressive drive gives a 
convincing description of the non-singularity of drive objects. While performing various kicks in Tae Kwon Do exercises, he found that his "...aggression could be turned on virtually any solid object, including any person who happened to be my assigned opponent."

Rapaport (1967) also recognized the non-singularity of drive objects, claiming that the assignment of object to drive in consequence of good fit for gratification implied "a coordination of the instinctual drive discharge with a definite (even if broad) range of objects, and an equifinality in regard to this range of objects" (p. 878). (Rapaport's assertion that the range is "definite" will not suffice to ensure determinancy. It does however suggest that he wanted this assurance too.) Rapaport (p. 878) further holds that that what constituted the range of well-fitted objects was likely "phylogenetically determined". Earlier (p. 833) he had noted a parallel between Freud's set of drive objects and the "releasers" in Lorenz's system of innate releasing mechanisms (the most well known of which is imprinting). Rapaport reports that Lorenz (1950) and Timbergen (1951) found that the releasers were not singular objects. Rather that they were "aggregates of stimuli, only some of which are indispensable for effecting adequate release, and various combinations of which can effect adequate release" (The quotation is from Rapaport 1967, p. 833).

This finding accords well with the above description of the object of Baby X's oral drive. This object was said to be any amalgam or part of the provider of satisfaction and/or the material object satisfying Baby $X$ 's oral drive: X's mother's left breast and nipple and her right breast and nipple and a bottle and nipple, and the milk, and her face, and how it feels being held and fed, and how her face and breast (or bottle) look, and the pacifier and the hand of its provider, and the blanket edge, especially how it feels in X's mouth - any part of this and all of it together.

Two things can be seen in all of these examples of drive objects: 1 . There is no primacy or originality of one part of these objects over another. In other words, displacement from some original is not taking place here for drive objects as it is for drive aims; 2 . These objects do not appear as singular and determinate, at least not in any ordinary way. Instead they are either amalgams of parts of persons or items (as in Baby $\mathrm{X}$ and in the animals studied by Lorenz and Timbergen); or a set of items linked as a set by some contingent feature as in Velleman's example of "any solid object" which happened to be available.

These mysterious objects can be understood however, once they are seen as being composed of elements comprising an a-rational primary 
process category. Primary process categories are collections grouped together, not at all randomly, but lawfully; not according to rational principles, but on the basis of the associative, a-rational principles of the primary process (see Freud 1900a, 1915e, 1926d, 1940a). Drive objects are constituted as such categories whose elements cohere owing to different primary process principles. For example, there is one type of primary process category held together due to a shared feature or attribute, one that need not be an essential feature. And indeed, there are drive objects which do seem to be constituted in just this way - take for example, Lorenz's ducklings who will imprint onto any moving item of a certain physical size, Velleman who would kick any solid object, and Baby $\mathrm{X}$ who sucks on anything that is available to suck. Another primary process category links things that are spatially contiguous. And we can see this sort of drive object too - i.e., Baby X's drive object consists of mother's left nipple and the milk, father's hand and the nipple on the bottle and the milk, the pacifier and the face of a parent, etc. (The topic of primary process categorizations has been addressed much more fully in Brakel 2002, 2003, 2004).

\section{4. CAN PRIMARY PROCESS OBJECTS BE REPRESENTED? HOW?}

Drive objects can be better understood when they are seen as objects that cohere on the basis of primary process principles. But there is still the problem of indeterminancy. How can such objects be represented? Given that the standard propositional-attitude psychology analysis used to individuate contentful representations of the objects of propositions will not work - and it will not work because drives are not standard propositional attitudes with singular, representable objects - is there some analysis that can fix the content of these objects?

Certainly fixing content with the normative assumption of interpretative holistic rationality (as do the attributionists, one group using propositional-attitude psychology), or applying any sort of rationality based fixing will not work. (For attributionist and other rationality based views, see especially Davidson 1980, 1984; Cherniak 1981; Stich 1983; and Dennett 1978, 1987.) How could this work when we are dealing with a-rational, albeit lawfully organized categories? What would work is an account with a different normative assumption than that of interpretable holistic rationality and yet an account not susceptible to the indeterminancy problem. These requirements can be met using the proper-function naturalism developed by Ruth Millikan (Millikan 1984, 
1993) in which the normative assumption of interpretative success and rational explanation is replaced with the normative assumption of selective success and evolutionary explanation.

I will briefly describe this fitness-based normativity. Then in keeping with the terms of this analysis, I will claim that indeed primary process based drive objects do have fixed, albeit non-singular contents insofar as they have a proper function, one which under certain conditions leads to fitness success. (What follows is an application of an argument concerning the proper function and fitness success of a-rational primary process phantasies, appearing in Brakel 2002).

\section{5. FITNESS BASED NORMATIVITY: A PROPER FUNCTION ACCOUNT OF REPRESENTATIONS OF ORDIANRY OBJECTS}

In order to demonstrate selective fitness normativity, let's start with something far from our area of concern - sweat glands. The function of sweat glands is to secrete sweat. But their "proper-function" entails what Millikan (1993) terms "Normal" conditions. Normal conditions are those in which properly functioning sweat glands contributed to the survival of animals possessing them and thereby increased the selective success of those animals. Thus sweat glands functioning flawlessly to produce sweat when there is a tumor at the temperature-sensing center are not performing their "proper-function" because they are not functioning under "Normal" conditions - i.e., those conditions contributing to the animal's selective reproductive advantage. Only those sweat glands producing sweat when the body is overheated are sweat glands properly-functioning under Normal conditions.

Now let's move closer to our area of interest, beliefs and their objects. Not all beliefs are properly-functioning under Normal conditions. Normal conditions, those which conferred selective fitness, are those in which the truth conditions of the representation (the object of belief) match those obtaining in the world. In other words only true beliefs are those properly-functioning under Normal conditions (Millikan 1993, p. 73). But note that in Millikan's program there is nothing a priori or necessary in the object of beliefs matching truth conditions in the world or in believer coming by the object of their beliefs rationally. Rather true beliefs and the rationality they usually entail are contingently determined biological norms. Yes, it happens to have been the case that our ancestors capable of having rationally mediated true beliefs, and exercising this capacity just frequently enough, had selective advantages such 
that the mechanisms supporting true-belief generation were reproduced. But that the Normal conditions for beliefs are those in which the truth conditions for the representations are constituted by the truth conditions of the world, is entirely a contingent matter. Note also that the Normal conditions need not be the typical conditions. Provided that there are enough Normal properly-functioning beliefs to fix content via fitness success and selective normativity, instances of abNormally functioning beliefs, i.e., false beliefs, incomplete beliefs, etc. will also have content.

\section{6. FITNESS BASED NORMATIVITY: A PROPER FUNCTION ACCOUNT OF AN UNUSUAL TYPE OF REPRESENTATION}

Closer still to our interest in primary process drive objects, let's turn to toads. Toads can be used to demonstrate 1. representational content without any rationality; 2 . abNormal instances that are nonetheless contentful; 3. representations which have fixed determinate content despite initial appearances to the contrary. Toads, Millikan (1993, p. 109) claims, have contentful representations of bugs, despite their incapacity to distinguish bugs from lead pellets. Toads have a powerful set of reflexes such that when bugs or lead pellets are within striking distance tongue protrusion, item capture, and item swallowing ensue in rapid sequence. Lots of bugs and lots of pellets are swallowed indiscriminately; and yet Millikan claims that toads have a determinate representation of bugs. Here is how. Although a toad cannot differentiate a bug from a lead pellet, presumably producing indistinguishable visual representations, only those representations which have represented actual bugs are the result of properly-functioning Normal condition representations. Why? Because only actual bugs have contributed to toad nutrition and thereby to toad reproductive success. And what about those representations that represent actual lead pellets? They are not properlyfunctioning Normal condition representations of bugs; nor are they any sort of representations of lead pellets; they are abNormal representation of bugs! The content "bug" is fixed in a determinate fashion according to selective fitness normativity.

\section{7. FITNESS BASED NORMATIVITY: A PROPER FUNCTION ACCOUNT OF PRIMARY PROCESS DRIVE OBJECTS}

A proper function account is given in two steps. First the proper function is set out; then the Normal or fitness enhancing conditions are 
proposed. So let's begin with the proper function for the object of drives.

\section{7. 1. Proper function}

Obviously, any proper function of the drive objects must ensure that the drive aims - both the aims specific to particular drives, and the ultimate aim of drive discharge - are satisfied, with the result that the demand for work the somatic source places on the mental apparatus is considerably diminished. Less obvious, but no less important, the proper function of drive objects constituted by primary process organized sets is two-fold. First the proper function is that such objects are constituted by elements connected in a primary process fashion. The resultant set is not in any way random, but coheres according to primary process principles - association by contiguity in time or space, and association by the similarity of inessential features. Second the proper function of such primary process drive objects allows that this sort of diversity of drive satisfaction is tolerated. Rapaport (1967, p. 833) suggests as much: "The coordination of instinctual drive and object is assumed to be innate, i.e., given by evolution. While this coordination is rigid in lower-order animals, it is relatively flexible in man." (The adaptational relations between drive, drive object, and environment is taken up in detail in Hartmann 1958). Rapaport continues by noting that there can be considerable "variability in the object of the instinctual drive". And finally Rapaport even challenges the rigidity of drive and drive object in animals. Citing the findings of Lorenz and Timbergen (quoted above) in which the so called "innate releasing mechanisms releasers" (drive object equivalents) are not singular and complete objects, but rather "aggregates of stimuli, only some of which are indispensable for effecting release, and various combinations of which can effect adequate release", Rapaport concludes: "The concept of releaser, like Freud's instinctualdrive object, implies some flexibility of the relation between the innate releasing mechanism and the releaser."

\section{7. 2. Normal conditions}

Having specified the proper function for drive objects constituted by primary process sets, the next step is to find those conditions under which the proper functioning of these drive objects could lead to fitness 
success. If found, these will be the Normal conditions, that allow the primary process drive objects to have fixed determinant content, without being univocal and singular. This is a surprisingly easy task. Let's look at the consequences if primary process amalgams or sets did not comprise drive objects. Suppose Lorenz's ducklings could imprint uniquely only onto their mothers. Yes, if you are a duckling it is ideal to imprint onto your mother; but suppose she is gone or sick? Isn't better to be able to imprint onto some other conspecifics (or even onto a researcher) and have a chance thereby to survive and reproduce? There seems to be clear selective fitness advantage in the diversity of the type of primary process organized "releasers" ducklings possess. Similarly with human babies, if Baby X's oral drives could only be quelled with mother's milk from her nipples, Baby $\mathrm{X}$ would likely be hungry more of the time, certainly discontent much of the time, and plausibly less likely to grow up healthy enough to reproduce. Jumping ahead developmentally, suppose drives from the Oedipal phase could have but a unique, singular type object. Wouldn't Oedipal phase resolution be even harder than it is? Wouldn't choices for mates be severely curtailed, at the detriment to both individual and species? If we turn to aggression, the same potential benefit for primary process objects can be seen. Suppose for some people the "aggressive drive" had as its object only conspecifics, those vying for a mate, those attacking first, etc. These people would be at a selective disadvantage with respect to nourishment to those whose aggressive drive also included prey and predator animals. The fitness disadvantage would be no less for people whose aggressive drive had the singular object of big predators. For these people would have trouble defending themselves and progeny against attacks from other people, as well as from noxious, but small animals (like snakes and spiders). They might also be at a calorie disadvantage, finding less aggressive drive pleasure in fishing and small game hunting.

In the last two subsections I have made a case for a proper function account of drive objects constituted by primary process amalgams or sets. There are criteria for the proper function of primary process set (or amalgam) based drive objects - namely, that the set of elements comprising the drive object is organized according to primary process principles; and that the resultant drive object allows diverse gratification. And there are Normal conditions under which the diversity facilitated by the primary process drive objects can enhance fitness success. Hence, the argument goes through; these primary process drive objects can be 
non-singular and yet have determinate content, with selective fitness normativity underwriting the constraint against indeterminancy.

\section{8. CONCLUDING REMARKS: FROG REPRESENTATIONS, VAGUE CONCEPTS, AND PRIMARY PROCESS DRIVE OBJECTS}

What is the relation of the representations associated with primary process drive objects, to those frogs have regarding bugs and BBs, and to those associated with "vague concepts", a current topic in philosophy? Williamson (1994) in his tour de force book, Vagueness, after an exhaustive review of all competing views, essentially concludes that vague concepts result from cognitive limitations. Vague concepts pick out something precise ontologically - there is a particular number of grains of sand at which point they will constitute a "heap"; there is a specific number of hairs on his head below which he will be bald - just as there is one and only one number that represents the precise number of people on Main Street in Ann Arbor right now - however our cognitive limitations do not let us know these precise numbers. (See p. 208, in particular). A similar case could be made for frogs' representations of bugs and BBs. Although they cannot distinguish bugs from BBs, and presumably represent them identically, this representation, on the proper function view I've been propounding, always refers to bugs - correctly when there is a bug to swallow, and incorrectly when a BB is ingested, but there is a correct representation. Yes frogs' representations are good enough; but if frogs were better cognizers, they could make the distinction and perhaps there would even be some selective advantage - fewer ingestings of non-nutritive item, which even if not harmful take up the time and space that could be devoted to real bugs.

Representations of primary process amalgamated drive objects, on the other hand, bear only a superficial relation to vague concepts - one perhaps based on an insufficient understanding of the nature of the primary process mental content in general and the nature of primary process drive objects in particular. Regarding primary process content itself, consider that intrinsically this content might be neither vague nor indeterminate; but only seem so relative to the secondary process terms in which we (as secondary process dominated thinkers) are forced to formulate it. Perhaps for any representational system (including that of the frogs and their bugs/BBs) there is in itself no vagueness or indeterminacy, but only the appearance of such when there is an attempt to translate it into a relatively more precise and determinate representational 
system. While the more precise and determinate second system is capable of the distinctions that the first system cannot make, the formulation or translation may not be workable, a situation which does not imply the vagueness or indeterminacy of the first system content.

Let's turn now to the nature of primary process drive objects. Indeed as I look at the crowd at University of Michigan football stadium, to me it seems equally likely that there are 99,546 people as 90,000 people as 98,765 people. The set of numbers comprising my representation is vague; and the elements I single out when I give a number are arbitrary and random. While it may seem to be similar that an unconstrained string of random and arbitrary elements can comprise a primary process drive object, as I have tried to show, this is not the case. Moreover, the amalgam or set must not only be derived according to its proper function, following the laws of primary process toward diversity in satisfaction, but is further constrained by the need for each element to be subjectively (if idiosyncratically) relevant to the individual with the drive. Thus my mother's face will not suffice to satisfy the oral drives of Baby $\mathrm{X}$; and the realm of possible Oedipal choices for me will not be the same as the realm for you. Further, improving cognitive capabilities would not seem to lead to the ability to constitute a "more correct" drive object, in the sense that without my human limitations I could more correctly know the number of hairs on Z's head below which would constitute baldness or the number of people at the $\mathrm{U}$ of $\mathrm{M}$ stadium. This owes to the fact that there is no correct or incorrect with respect to this primary process amalgam. But would fewer cognitive limitations allow "better" (if not more correct) drive objects, objects that fitted better the environment, toward greater satisfaction and ultimately enhanced fitness success? It is tempting to answer yes; but at the same time it is hard to imagine any rational matching process being as resiliently successful in providing drive and drive object fit, as are the a-rational primary process drive object amalgams, with their built in diversity. 


\section{REFERENCES}

Brakel, L. (2002). Phantasy and wish: A proper function account of a-rational primary process mediated mentation. Australasian Journal of Philosophy 80: $1-16$.

Brakel, L. (2003). "Unusual" human experiences: Kant, Freud and an associationist law. Theoria et Historia Scientiarum 7(2): 109-116.

Brakel, L. (2004). Der Primärvorgang: Experimentalle Belege für A-rationale Organisationsprinzipien und einen philosophischen Fall von A-rationaler Referenz. In Psychoanalyse im Dialog der Wissenschaften. Vol. 2. Angloamerikanische Perspektiven, ed. P. Giampieri-Deutsch. Stuttgart: Kohlhammer, pp. 143-167.

Cherniak, R. (1981). Minimal rationality. Mind 90: 161-183.

Davidson, D. (1980). Actions and Events. Oxford: Clarendon Press.

Davidson, D. (1984). Truth and Interpretation. Oxford: Clarendon Press.

Dennett, D. (1978). Brainstorms. Cambridge, Mass.: MIT Press.

Dennett, D. (1987). The Intentional Stance. Cambridge, Mass.: MIT Press.

Erikson, E. (1950). Childhood and Society. New York: Norton.

Freud, A. (1936). The Ego and the Mechanisms of Defense. In The Writings of Anna Freud. Vol. 2. New York: International Universities Press 1966.

Freud, A. (1977). A study guide to Freud's Writings. In The Writings of Anna Freud. Vol. 8. New York: International Universities Press 1981, pp. 209-276.

Freud, S. (1900a). The Interpretation of Dreams. S.E. 4-5.

Freud, S. (1905e). A case of hysteria. S.E. 7: 7-122.

Freud, S. (1905d). Three Essays on Sexuality. S.E. 7: 135-243.

Freud, S. (1909b). Analysis of a phobia in a five year old boy. S.E. 10: 5-147.

Freud, S. (1909d). Notes upon a case of obsessional neurosis. S.E. 10: 155-249.

Freud, S. (1915c). Instincts and their vicissitudes. S.E. 14: 117-140.

Freud, S. (1915e). The unconscious. S.E. 14: 166-204.

Freud, S. (1918b). An infantile neurosis. S.E. 17: 7-122.

Freud, S. (1926d). Inhibitions Symptoms and Anxiety. S.E. 20: 87-172.

Freud, S. (1940a). An Outline of Psycho-Analysis. S.E. 23:144-207.

Hartmann, H. (1958). Ego psychology and the problem of adaptation. New York: International Universities Press.

Lorenz, K. (1950). The comparative method in studying innate bird behavior patterns. In Physiological Mechanisms in Animal Behavior, Symposia of the Society for Experimental Biology. New York: Academic Press.

Millikan, R. (1984). Language, Thought, and Other Biological Categories. Cambridge, Mass.: MIT Press.

Millikan, R. (1993). White Queen Psychology and Other Essays for Alice. Cambridge, Mass.: MIT Press.

Nagera, H. (1969). Basic Psychoanalytic Concepts on the Libido Theory. Vol. 1. New York: Basic Books.

Nagera, H. (1970). Basic Psychoanalytic Concepts on the Theory of Instincts. Vol. 3. New York: Basic Books.

Rapaport, D. (1967). The Collected Papers of David Rapaport. New York: Basic Books. 
Stich, S. (1983). From Folk Psychology to Cognitive Science: The Case Against Belief. Cambridge, Mass.: MIT Press.

Timbergen, N. (1951). The Study of Instinct. Oxford: Clarendon Press.

Velleman, D. (2002). Motivation by ideal. Philosophical Explorations 5: 89-104.

Williamson, T. (1994). Vagueness. London and New York: Routledge. 